\title{
FIFA Player Ranking Based on Tweets Recognizing Named Entity
}

\author{
Md. Niaz Imtiaz ${ }^{1}$, Md Toukir Ahmed ${ }^{1}$, and Golum Rabby ${ }^{1}$ \\ ${ }^{1}$ Pabna University of Science and Technology
}

June 13, 2020

\begin{abstract}
Nowadays social media play an important role to share opinions on various events. People often share their experiences, opinions and measurements. Social media have become important sources of information to evaluate different types of events and people as well. Twitter has become one of the popular social media platforms to express news in real-time. Opinions shared on social media can create impacts on various events. People often share their opinions and feelings about players. Football is one of the most popular game all around the world. People love to watch football, analyze players and post their opinions on social media. In this thesis, an analysis is performed on FIFA top listed eight players of the current time, based on tweets of different persons. 5000 tweets are extracted and by applying the Named Entity Recognition technique tweets on each player are isolated. A sentiment analysis approach is applied on tweets to measure the polarity of each tweet. A ranking system is designed to rank the players. By different computational and statistical means and using the designed ranking system the chosen players are ranked. A comparative analysis among FIFA best eight players is shown as well according to public views on them.
\end{abstract}

\section{Hosted file}

ENG-2020-06-0427.pdf available at https://authorea.com/users/333235/articles/459457-fifaplayer-ranking-based-on-tweets-recognizing-named-entity 